\title{
V-BLAST Outage Probability: Analytical Analysis
}

\author{
Sergey Loyka \\ School of Information Technology and Engineering (SITE) \\ University of Ottawa, 161 Louis Pasteur, Ottawa, Ontario, Canada, K1N 6N5 \\ Email: sergey.loyka@ieee.org
}

\begin{abstract}
An analytical approach to the performance analysis of the V-BLAST algorithm is discussed in this paper. It is based on closed-form analytical models of the three key algorithm components: interference cancellation, interference nulling and optimal ordering. The closed-form analytical model of the GramSchmidt orthogonalization process is a key component of the proposed analysis method. It allows to derive closed-form expressions for signal at each detection step, to perform analytically statistical analysis for a Rayleigh-fading channel (i.e., diversity order etc.) and to obtain closed-form expressions for outage probabilities in the case of two $T x$ antennas. In particular, it is demonstrated that the optimal ordering does not result in increased diversity order, but only in a fixed SNR gain. Generalized versions of the V-BLAST algorithm proposed recently can be analyzed in a similar way.
\end{abstract}

Keywords-MIMO, V-BLAST, multi-antenna system, fading, outage probability, BER

\section{INTRODUCTION}

Information-theoretic considerations show that the multiple-input multiple-output (MIMO) communication architecture is able to provide extraordinary high spectral efficiencies in rich multipath environments [1-3]. Space-time coding and/or a special signal processing algorithm is to be implemented at the receiver in order to achieve at least part of the MIMO channel capacity. Diagonal Bell Labs Layered Space-Time (D-BLAST) algorithm has been proposed by Foschini for this purpose, which is capable of achieving a substantial part of the MIMO capacity [1]. However, a high complexity of the algorithm implementation is its substantial drawback. A simplified version of the BLAST algorithm is known as V-BLAST (vertical BLAST). It is capable of achieving high spectral efficiency while being relatively simple to implement [4].

Comprehensive evaluation of the system performance is required because the matrix wireless propagation channel may severely degrade the performance of this algorithm. Some preliminary studies including asymptotic analysis and numerical Monte-Carlo simulations have been reported in [5]. While the numerical Monte-Carlo approach is useful from many viewpoints, the analytical approach provides deeper insight and comprehensive understanding of the key points in the algorithm operation. In particular, as we demonstrate in the paper, closed-form analytical expressions for outage probability (cumulative distribution function) at each detection step can be derived and, consequently, closed-form analytical expressions for BER can also be obtained in some cases.

In this paper, we develop a unified analytical approach to the analysis of the V-BLAST algorithm operation based on some general geometrical ideas presented in [6]. The approach is based on the closed-form analytical models of the key VBLAST and associated system components - interference nulling from yet to be detected symbols (Gram-Schmidt orthogonalization process), interference subtraction from already detected symbols, the optimal ordering procedure (based on the after processing SNR), optimal (maximum ratio or similar) combining, and a statistical (complex circular Gaussian) model of the matrix wireless propagation channel. In particular, closed-form analytical expressions for the signal and noise vectors at each processing step are derived for wireless channel with the general correlation matrix. Based on these results, a rigorous proof that the diversity order at the $i$-th processing step is $n-m+i$ (where $n$ and $m$ are the number of $\mathrm{Rx}$ and Tx antennas correspondingly) is given for uncorrelated Rayleigh channel and if no optimal ordering is used. While the previous result is valid in general $n x m$ case, we are able to analyze analytically the outage probability when the optimal ordering is implemented for $m=2$ only due to mathematical difficulties arising in general case. We show that the optimal ordering results in increasing the after processing SNR by 3 dBs rather than to increase the diversity order (as one would intuitively expect based on the selection combining argument) at the first detection step. At the second step, the effect of optimal ordering is to increase the outage probability twice (or to decrease the after-processing SNR). The diversity order is $n$ at this step. Closed-form analytical expressions are obtained for outage probabilities (cumulative distribution functions) at each detection step. Hence, BER expressions can be further derived using these results and one of the well-known methods. The analytical results above are verified using extensive numerical Monte-Carlo simulations.

\section{V-BLAST ALGORITHM}

The V-BLAST algorithm has been discussed in details elsewhere [4,5]. Here we describe its main points for completeness and in order to introduce notations. The main idea of the BLAST architecture is to split the information bit stream into several sub-streams and transmit them in parallel using a set of Tx antennas (the number of Tx antennas equals the number of sub-streams) at the same time and frequency. At the $\mathrm{Rx}$ side, each $\mathrm{Rx}$ antennas "sees" all the transmitted signals, which are mixed due to the nature of the wireless propagation channel. Using appropriate signal processing at the Rx side, these signals can be unmixed so that the matrix wireless channel is transformed into a set of virtual parallel independent channels (provided that mutltipath is rich enough).

The following basic assumptions are employed:

- The channel is random, quasistatic (i.e. fixed for every frame of information bits but varying from frame to frame), frequency independent (i.e., negligible delay spread) and with complex AWGN. 
- The Tx signal vector is comprised of individual symbol sub-streams. No space-time coding is employed.

- The noise vector is comprised of independent AWGN components with equal variance.

- The Tx signals, noise and channel gains are independent of each other

- Perfect channel knowledge is assumed to be available at the receiver.

- There is no performance degradation due to synchronization and timing errors.

The received signal vector $\mathbf{r}$ can be presented in the following complex baseband vector form [5]:

$$
\mathbf{r}=\mathbf{H q}+\mathbf{v}=\sum_{i} \mathbf{h}_{i} q_{i}+v
$$

where $\mathbf{q}=\left[\begin{array}{lll}q_{1} & \ldots & q_{m}\end{array}\right]^{\mathrm{T}}$ is the transmitted symbol vector, $\mathbf{H}$ is the channel matrix (i.e., the matrix of complex transfer factors from each Tx to each Rx antenna), $\mathbf{v}=\left[\begin{array}{lll}v_{1} & \ldots & v_{n}\end{array}\right]^{\mathrm{T}}$ is the noise vector and $\mathbf{H}=\left[\begin{array}{lll}\mathbf{h}_{1} & \ldots & \mathbf{h}_{m}\end{array}\right]$, where $\mathbf{h}_{\mathrm{i}}$ is a column vector of transfer factors from $\mathrm{i}$-th $\mathrm{Tx}$ antenna to all $\mathrm{Rx}$ antennas.

The V-BLAST processing begins with the $1^{\text {st }}$ Tx symbol and proceeds in sequence to the $\mathrm{m}$-th symbol. When the optimal ordering procedure is employed, the Tx indexing is changed prior to the processing. The main steps of the VBLAST processing (detection) algorithm are as follows [4,5]:

The interference cancellation step: at the $\mathrm{i}$-th processing step (i.e., when the signal from the i-th transmitter is detected) the interference from the first i- 1 transmitters can be subtracted based on the estimations of the Tx symbols (which are actually assumed to be error-free) and the knowledge of $\mathbf{H}$.

The interference nulling step: based on the knowledge of the channel matrix, the interference from yet to be detected symbols can be nulled out using the Gram-Schmidt orthogonalization process (applied to the column vectors of H).

The optimal ordering procedure: the order of symbol processing is organized according to their after-processing SNRs in the decreasing order (i.e., the symbol with highest SNR is detected first).

\section{ANALYSIS OF THE V-BLAST ALGORITHM}

For completeness, we summarize here the main results of the V-BLAST algorithm analysis presented in [6]. For the sake of notational simplicity, we first describe all the steps without the noise contribution $(v=0)$, which is added to the analysis later.

The interference cancellation step can be expressed mathematically in a straightforward way [5]. The received signal after the cancellation at the i-th step is:

$$
\mathbf{r}_{i}^{\prime}=\mathbf{r}-\sum_{j=1}^{i-1} \mathbf{h}_{j} \hat{q}_{j}
$$

where $\hat{q}_{j}$ are the estimations of the already-detected symbols.

In the further analysis, we assume for the sake of simplicity that they are error-free. This is a valid assumption in low BER (high SNR) mode [5] (the effect of estimation errors is a second-order one). It should be noted that the analysis below can be modified in a straightforward way to account for the estimation errors.
The interference nulling step is based on the Gram-Schmidt ortogonalization procedure, which builds a set of orthogonal vectors from a set of linearly-independent vectors. At this stage, we assume that $\mathbf{h}_{\mathrm{i}}$ are linearly independent (otherwise the V-BLAST algorithm must be modified taking into account all the linearly dependent column vectors and decreasing the number of independent bit sub-streams). Using the closed from analytical expression for the Gram-Schmidt process and after some mathematical development (see [6] for details), we arrive to the following expression of the received vector after interference nulling out and cancellation at the i-th step:

$$
\mathbf{r}_{i}{ }^{\prime \prime}=\frac{q_{i}\left|\mathbf{h}_{i}\right|}{\left|\mathbf{R}^{[i+1, m]}\right|}\left|\begin{array}{cccc}
\eta_{i} & \eta_{i} \eta_{i+1} & \ldots & \eta_{i} \eta_{m} \\
\eta_{i+1} & & & \\
\ldots & & \mathbf{R}^{[i+1, m]} & \\
\eta_{m} & &
\end{array}\right|
$$

where $\left|\mathbf{h}_{i}\right|=\sqrt{\sum_{j}\left|h_{j i}\right|^{2}},||$ means determinant when applied to a matrix, $\quad \eta_{i}=\mathbf{h}_{i} /\left|\mathbf{h}_{i}\right|, \quad \eta_{i} \eta_{j}=\sum_{k=1}^{n} \eta_{k i} \eta_{k j}{ }^{*}, *$ denotes complex conjugate, and $\mathbf{R}^{[i+1, m]}$ is the normalized instantaneous (i.e., for a given channel realization) channel correlation matrix built on $\left[\begin{array}{lll}\eta_{i+1} & \ldots & \eta_{m}\end{array}\right]: \mathbf{R}_{k l}^{[i+1, m]}=\eta_{i+k} \eta_{i+l}$, $k, l=1, \ldots, m-i$. The signal power can be expressed as [6]:

$$
\left|\mathbf{r}_{i}^{\prime \prime}\right|^{2}=\left|q_{i}\right|^{2}\left|\mathbf{h}_{i}\right|^{2}\left|\mathbf{R}^{[i, m]}\right|\left|\mathbf{R}^{[i+1, m]}\right|^{-1}
$$

From this result and using (6), it is straightforward to obtain a bit error rate for a particular modulation scheme.

Let us consider the optimal ordering at the 1-st step for arbitrary $m$. When the $\mathrm{i}$-th Tx symbol is detected first, the signal power after interference nulling out is:

$$
P_{i}=\left|q_{i}\right|^{2}\left|\mathbf{h}_{i}\right|^{2}|\mathbf{R}|\left|\mathbf{R}^{[i]}\right|^{-1}
$$

where $\mathbf{R}$ is the full correlation matrix (i.e., built on $\left.\left[\begin{array}{lll}\eta_{1} & \ldots & \eta_{m}\end{array}\right]\right)$ and $\mathbf{R}^{[i]}$ is the correlation matrix built on all column vectors except for $\eta_{i}$. Under the assumptions of equal $\left|q_{i}\right|$ and equal $\left|\mathbf{h}_{i}\right|$ (i.e., the same received power from every transmit antenna), the optimal ordering is to detect first the symbol with the smallest $\left|\mathbf{R}^{[i]}\right|$. In fact, this means that the

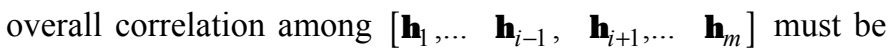
highest and, consequently, the correlation between $\eta_{i}$ and $\left[\begin{array}{llll}\eta_{1}, \ldots & \eta_{i-1}, & \eta_{i+1}, \ldots & \eta_{m}\end{array}\right]$ must be the lowest (this follows from geometrical interpretation of $|\mathbf{R}|$ as a volume in the mdimensional space [7]). Thus, the best ordering is to detect first that symbol whose column propagation vector has lowest correlation with the other vectors.

The after-processing noise power at i-th step can be simply expressed as [6]:

$$
P_{v_{i}}=\left\langle\left|v_{i}\right|^{2}\right\rangle=(n-m+i) \sigma_{1}^{2}
$$

where $\sigma_{1}^{2}=\left\langle\left|v_{j}\right|^{2}\right\rangle$ is per-branch noise power before processing, and \langle\rangle is the expectation over noise voltage (see 
[6] for detailed derivation). Note that the after-processing noise power is less than the total noise power, which is $n \sigma_{1}^{2}$. This is the consequence of the orthogonal projection performed by the Gram-Schmidt process (see Fig. 1). One also should note that the after-processing noise power increases with $i$ (step index), being the smallest in the $1^{\text {st }}$ step and the same as the total noise power in the last step.

\section{FADING OUTAGE CURVES AND DIVERSITY ORDER}

Based on the results above, let us know analyze the signal fading in the V-BLAST system. In particular, we consider the outage probabilities (i.e., the probability that the signal level is less than the specified value) and diversity order (i.e., the asymptotic slope of the outage probability curve).

We assume that the channel gains (i.e., the components of H) are i.i.d. complex Gaussians with zero mean and unit variance (i.e., we consider only the channel variation due to multipath and ignore the absolute propagation loss and largescale variation due to shadowing). First, we ignore the optimal ordering procedure and prove that the diversity order at the $i$-th step is $(n-m+i)$.

To demonstrate the main idea of the proof, let us consider first the case of $n=m=2$, i.e. $\mathbf{H}=\left[\begin{array}{ll}\mathbf{h}_{1} & \mathbf{h}_{2}\end{array}\right]$. To be specific, we assume that the 1-st $\mathrm{Tx}$ symbol is detected first. The interference nulling out can be expressed is a general matrix form: $\mathbf{r}_{\perp}=Q \cdot \mathbf{r}$, where $Q$ is an orthogonal projection matrix, which projects $\mathbf{r}$ to the direction orthogonal to $\mathbf{h}_{2}$. Substituting this into (2), one obtains (since we are interested in the received signal power only, we ignore noise in this section): $\mathbf{r}_{\perp}=q_{1} Q \cdot \mathbf{h}_{1}$. This means that the signal after interference nulling out is proportional to that part of $\mathbf{h}_{1}$ which is orthogonal to $\mathbf{h}_{2}$, see Fig. 1, and the signal power $\sim\left|\mathbf{h}_{1 \perp}\right|^{2}$. But the vector magnitude is not affected by rotation on an arbitrary angle. We rotate $\left[\begin{array}{ll}\mathbf{h}_{1} & \mathbf{h}_{2}\end{array}\right]$ as a whole on angle $\psi$ so that $\mathbf{h}_{2}$ is parallel to $\mathbf{e}_{2}: h_{1,2}=0$. This can be expressed as: $\tilde{\mathbf{h}}_{i}=A \cdot \mathbf{h}_{i}$, where $A$ is the rotation matrix, which satisfies to (preservation of length): $A \cdot A^{+}=A^{+} \cdot A=I$, where "+" denotes conjugate transpose. Using the expressions above, one obtains: $\left|\mathbf{h}_{1 \perp}\right|=\left|\tilde{h}_{1,1}\right|$. It is further straightforward to show that the components of $\tilde{\mathbf{h}}_{1}$ has the same distribution as the components of $\mathbf{h}_{1}$ (note that $\psi$ is independent of $\mathbf{h}_{1}$ ), i.e. i.i.d. complex Gaussians with unit variance. Hence, $\left|\mathbf{h}_{1 \perp}\right|^{2}$ is chi-squared random variable with two degrees of freedom, $\left|\mathbf{h}_{1 \perp}\right|^{2} \sim \chi_{2}^{2}$. The same is true for the signal power. Thus, the diversity order in the 1-st step is one. The similar consideration for arbitrary $n$ leads to the conclusion that $\left|\mathbf{h}_{1 \perp}\right|^{2} \sim \chi_{2(n-1)}^{2}$ (simply because $\mathbf{h}_{1 \perp}$ has $n-1$ non-zero components after rotation) and the diversity order is $(n-1)$.

The case of arbitrary $m$ is somewhat more complex however straightforward to consider in the similar way. First, we rotate the set $\left[\begin{array}{lll}\mathbf{h}_{1} & \ldots & \mathbf{h}_{m}\end{array}\right]$ as a whole so that $\mathbf{h}_{m}$ becomes parallel to $\mathbf{e}_{m}$. In the second rotation we keep $\mathbf{h}_{m}$ fixed (i.e., a rotation around $\mathbf{e}_{m}$ axis) and position $\mathbf{h}_{m-1}$ into $\left[\begin{array}{ll}\mathbf{e}_{m-1} & \mathbf{e}_{m}\end{array}\right]$ plane. The rotations are continued until $\mathbf{h}_{2}$ is positioned into $\left[\begin{array}{lll}\mathbf{e}_{2} \mathbf{e}_{3} & \ldots & \mathbf{e}_{m}\end{array}\right]$ hypeplane. After the rotation, $\mathbf{h}_{1 \perp}$ has $(n-m+1)$ non-zero components. Every such rotation preserves the distribution of the components. Hence, $\left|\mathbf{h}_{1 \perp}\right|^{2} \sim \chi_{2(n-m+1)}^{2}$ and the diversity order is $(n-m+1)$. Similar consideration for the ith step leads to the conclusion that $\left|\mathbf{h}_{i \perp}\right|^{2} \sim \chi_{2(n-m+i)}^{2}$ and the diversity order is $(n-m+i)$. Note that the lowest diversity order is at the 1-st step and the highest is at the last (i.e., $n$ ). When $n=m$, no diversity is obtained at the 1 -st step.

\section{THE EFFECT OF OPTIMAL ORDERING}

For the sake of simplicity, we assume that all transmitted symbols have the same unit power and that the channel coefficients are i.i.d. complex Gausians with unit variance and zero mean (i.e. Rayleigh fading). Since we are interested in outage probability (i.e., the probability that the received signal power drops below a given value), we consider the received powers only (in each Rx antenna from each Tx antenna). Under the assumptions above, the vector signal received by the $\mathrm{Rx}$ antennas from the $\mathrm{i}$-th Tx antenna is $\mathbf{r}_{i}=\mathbf{h}_{i}$. We consider first $2 \times 2$ V-BLAST and further it is generalized to the $2 \times n$ case.

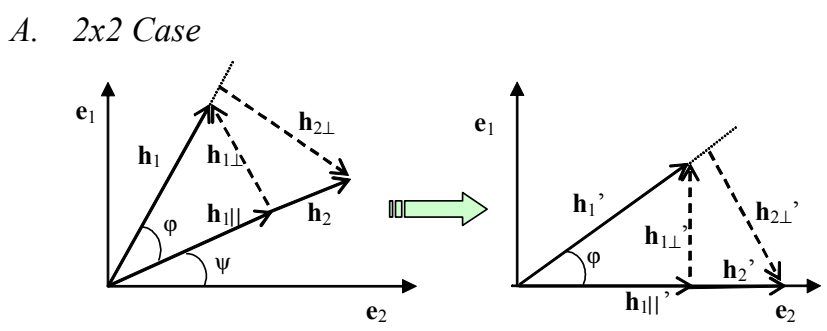

Figure 1. Rotation of the received vectors by angle $\Psi$.

Consider the following transformation of the received vectors (as a whole) illustrated in Fig. 1. Since it is the rotation, the vector lengths (i.e., the signal magnitudes) as well as the angle $\varphi$ are not changed. This rotation is the same as the one in the previous section. Hence, the primed vector lengths have the same distribution as the unprimed ones, namely $\left|\mathbf{h}_{1 \perp}\right|^{2} \sim \chi_{2}^{2}, \mathbf{h}_{1 \|} \sim \chi_{2}^{2},\left|\mathbf{h}_{1}\right|^{2} \sim \chi_{4}^{2}$. Thus, we further use unprimed notations. The components of $\mathbf{h}_{2}$ have the same distributions as those of $\mathbf{h}_{1}$. The outage probability (i.e. cumulative distribution function) for $\left|\mathbf{h}_{1}\right|^{2}$ (or $\left|\mathbf{h}_{2}\right|^{2}$ ) is

$$
\operatorname{Pr}\left[\left|\mathbf{h}_{1}\right|^{2}<x\right]=\operatorname{Pr}\left[\left|\mathbf{h}_{2}\right|^{2}<x\right]=F_{h}(x)=1-e^{-x}(1+x)
$$

i.e., the 2 nd order MRC. The optimal ordering procedure (after the interference nulling) can be described as follows:

$$
s_{1}=\max \left[\left|\mathbf{h}_{1 \perp}\right|^{2},\left|\mathbf{h}_{2 \perp}\right|^{2}\right]=(\sin \varphi)^{2} \max \left[\left|\mathbf{h}_{1}\right|^{2},\left|\mathbf{h}_{2}\right|^{2}\right]
$$


where $s_{1}$ is the signal power after the optimal ordering, i.e. we compare $\left|\mathbf{h}_{1 \perp}\right|$ and $\left|\mathbf{h}_{2 \perp}\right|$ and take the maximum. In fact, (8) tells us that the optimal ordering for $2 \times 2$ system is to detect first the sub-stream with the largest before-detection power (i.e., the max at the right-hand side of (8)). While $\left|\mathbf{h}_{1 \perp}\right|^{2}$ and $\left|\mathbf{h}_{2 \perp}\right|^{2}$ are $\chi_{2}^{2}$ (i.e., Rayleigh distributed), taking the maximum does not result in $2^{\text {nd }}$-order diversity because they are not independent, as $\sin \varphi$ at the right-hand side of (8) indicates. Using the fact that the distribution of $\max \left[\left|\mathbf{h}_{1}\right|^{2},\left|\mathbf{h}_{2}\right|^{2}\right]$ is $F_{h}^{2}(x)$, the distribution $F_{1}(x)$ of $\mathrm{s}_{1}$ may be presented in the following form:

$$
F_{1}(x)=\int_{0}^{\pi / 2} F_{h}^{2}\left(\frac{x}{\sin ^{2} \varphi}\right) f_{\varphi}(\varphi) d \varphi
$$

where $f_{\varphi}(\varphi)$ is the p.d.f. of $\varphi$ (note that it is not uniform). It can be shown that $f_{\varphi}(\varphi)=\sin 2 \varphi$. This p.d.f. is illustrated in Fig. 2. The most probable direction is $45^{\circ}$ as one would intuitively expect (because $\left|\mathbf{h}_{1 \perp}\right|^{2} \sim \chi_{2}^{2},\left|\mathbf{h}_{1 \|}\right|^{2} \sim \chi_{2}^{2}$ and, hence, the most probable values of the abscissa $\left(\left|\mathbf{h}_{1 \|}\right|\right)$ and ordinate $\left(\left|\mathbf{h}_{1 \perp}\right|\right)$ are the same (see Fig. 1)). The probability to get the angle close to $0^{0}\left(90^{0}\right)$ is very small because it means that $\left|\mathbf{h}_{1 \|}\right|$ $\left(\left|\mathbf{h}_{1 \perp}\right|\right)$ can be any and $\left|\mathbf{h}_{1 \perp}\right|\left(\left|\mathbf{h}_{1||}\right|\right)$ must be close to 0 , and this probability is small.

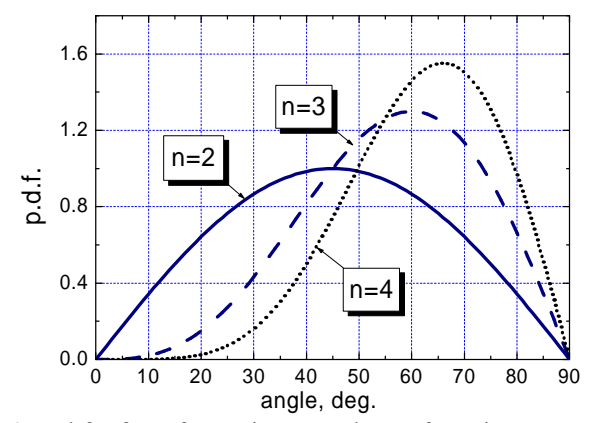

Figure 2. p.d.f. of $\varphi$ for various numbers of receive antennas (n).

It should be noted that (9) holds true in the $2 \mathrm{xn}$ case as well, provided that the appropriate expressions are used for $f_{\varphi}(\varphi)$ and $F_{h}(x)$. Evaluating the integral in (9), one obtains, after some manipulations, the following simple expression for the outage probability at the $1^{\text {st }}$ detection step with the optimal ordering:

$$
F_{1}(x)=1-2 e^{-x}+\left(1+\frac{x}{2}\right) e^{-2 x}
$$

The asymptotic behavior of this outage probability (i.e., in the small outage probability region) is

$$
F_{1}(x) \approx x / 2, \quad x \rightarrow 0
$$

Comparing this with the asymptotic behavior of the Rayleigh distribution $\left(F_{R}(x) \approx x, \quad x \rightarrow 0\right)$, we conclude that the effect of optimal ordering is to increase SNR (or decrease outage probability) by $3 \mathrm{~dB}$ rather than to increase the diversity order, as one might intuitively expect based on the selection combining argument (see (8)). The reason for this is that $\left|\mathbf{h}_{1 \perp}\right|$ and $\left|\mathbf{h}_{2 \perp}\right|$ are correlated.

The outage probability at the $2^{\text {nd }}$ detection step can be derived using an expression similar to (8). In particular, we note that at the second step we have to use the received vector with the minimum length $\min \left[\left|\mathbf{h}_{1}\right|,\left|\mathbf{h}_{2}\right|\right]$ because the vector with the maximum length was used in the $1^{\text {st }}$ step. We also note that there is no need for interference nulling at this step. Hence, $s_{2}=\min \left[\left|\mathbf{h}_{1}\right|^{2},\left|\mathbf{h}_{2}\right|^{2}\right]$ and the outage probability $F_{2}(x)$ is

$$
F_{2}(x)=\operatorname{Pr}\left[s_{2}<x\right]=F_{h}(x)\left[2-F_{h}(x)\right]
$$

Its asymptotic behavior is

$$
F_{2}(x) \approx 2 F_{h}(x) \approx x^{2}, \quad x \rightarrow 0
$$

Comparing it with the $2^{\text {nd }}$ order MRC outage probability, $F_{M R C}(x)=F_{h}(x) \approx x^{2} / 2$, we conclude that the effect of optimal ordering at the $2^{\text {nd }}$ detection step is to increase outage probability twice $(3 \mathrm{~dB})$. This is the "price" one has to pay for the $3 \mathrm{~dB}$ increase in SNR at the $1^{\text {st }}$ step. It should be noted that (12) holds true in the $2 \times n$ case as well, provided that the appropriate expression is used for $F_{h}(x)$.

\section{B. 2xn Case}

In this case, $\left|\mathbf{h}_{1 \perp}\right|^{2} \sim \chi_{2 n-2}^{2}, \quad \mathbf{h}_{1 \|} \sim \chi_{2}^{2}, \quad\left|\mathbf{h}_{1}\right|^{2} \sim \chi_{2 n}^{2}$ and

$$
F_{h}(x)=\operatorname{Pr}\left[\left|\mathbf{h}_{1}\right|^{2}<x\right]=1-e^{-x} \sum_{k=0}^{n-1} \frac{x^{k}}{k !}
$$

The same distributions hold true for $\mathbf{h}_{2}$ as well. It can be shown that in this case $f_{\varphi}(\varphi)=2(n-1)(\sin \varphi)^{2 n-3} \cos \varphi$. This p.d.f. is illustrated in Fig. 2 for $n=2,3$, 4. The most probable direction shifts to the right $\left(90^{\circ}\right)$ when $n$ increases, starting from $45^{\circ}$ for $n=2$. This is what should be intuitively expected because $\mathbf{h}_{1 \|} \sim \chi_{2}^{2}$ and $\left|\mathbf{h}_{1 \perp}\right|^{2} \sim \chi_{2 n-2}^{2}$, and, hence, the most probable value of the ordinate $\left(\left|\mathbf{h}_{1 \perp}\right|\right)$ is greater than the most probable value of the abscissa $\left(\left|\mathbf{h}_{1 \|}\right|\right.$ ) (for $\left.n>2\right)$, and the former increases with $n$ while the later is fixed. Using (9) and the p.d.f. above, one obtains,

$$
F_{1}(x)=\int_{0}^{\pi / 2} F_{h}^{2}\left(\frac{x}{\sin ^{2} \varphi}\right) f_{\varphi}(\varphi) d \varphi=(n-1) \int_{0}^{1} F_{h}^{2}\left(\frac{x}{t}\right) \cdot t^{n-2} d t
$$

After some manipulations, (15) reduces to,

$$
F_{1}(x)=1-(n-1) e^{-x}\left[\sum_{i=0}^{n-2}\left(2 a_{i}-e^{-x}\left[b_{i}+(2 x)^{n-1} c_{i}\right]\right) x^{i}\right]
$$

where 


$$
\begin{aligned}
a_{i} & =\sum_{m=0}^{i} \frac{(-1)^{i-m}(n-i-2) !}{(n-m-1) ! m !}, b_{i}=(-2)^{i}(n-i-2) ! \sum_{k=0}^{i} \frac{(-1)^{k}}{k !(n-1-k) !} \\
c_{i} & =\frac{2^{i}}{i !} \sum_{j=i+n}^{2 n-2} \sum_{k=j-n+1}^{n-2} \frac{(j-n) ! 2^{-j}}{k !(j-k) !}
\end{aligned}
$$

Hence, a general form of $F_{l}(x)$ is

$$
F_{1}(x)=1-p_{1}(x) e^{-x}+p_{2}(x) e^{-2 x}
$$

where $p_{1}(x)$ and $p_{2}(x)$ are polynomials of degree at most $(n$ $2)$ and $(2 n-3)$ correspondingly. The asymptotic behavior of the outage probability is

$$
F_{1}(x) \approx \frac{1}{(n-1) !}\left(\frac{x}{2}\right)^{n-1}, x \rightarrow 0
$$

Comparing it with (n-1)-order MRC asymptotic behavior, $F_{M R C}(x) \approx x^{n-1} /(n-1)$ !, we conclude that the effect of the optimal ordering at the $1^{\text {st }}$ detection step is to increase SNR by $3 \mathrm{~dB}$ rather than to increase the diversity order. It is interesting to note that the conclusion proved to be true for $2 \times 2$ system, is also true in the general $2 \mathrm{x} n$ case. The outage probability at the $2^{\text {nd }}$ detection step is given by (12), where $F_{h}(x)$ is that in (14). We do the same conclusion as in the $2 \times 2$ case: the effect of optimal ordering at the $2^{\text {nd }}$ detection step is to increase the outage probability twice. This is the "price" to pay for the increased SNR at the $1^{\text {st }}$ step.

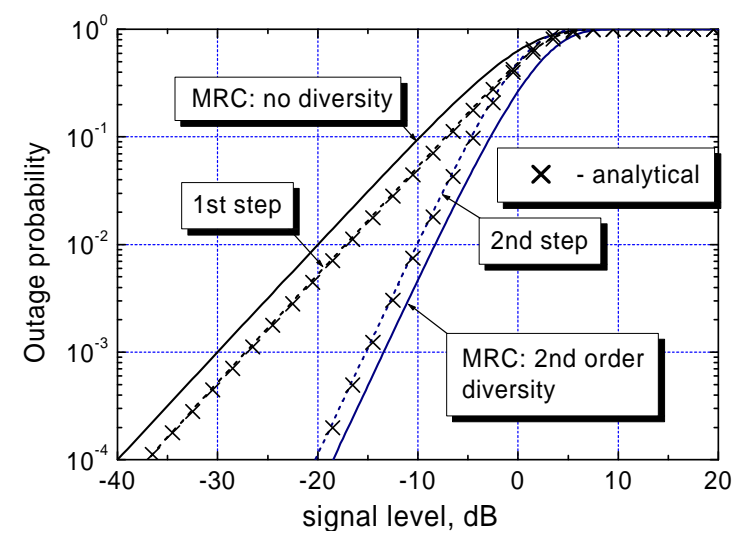

Figure 3. Outage probability curves of the V-BLAST algorithm for $\mathrm{n}=2$.

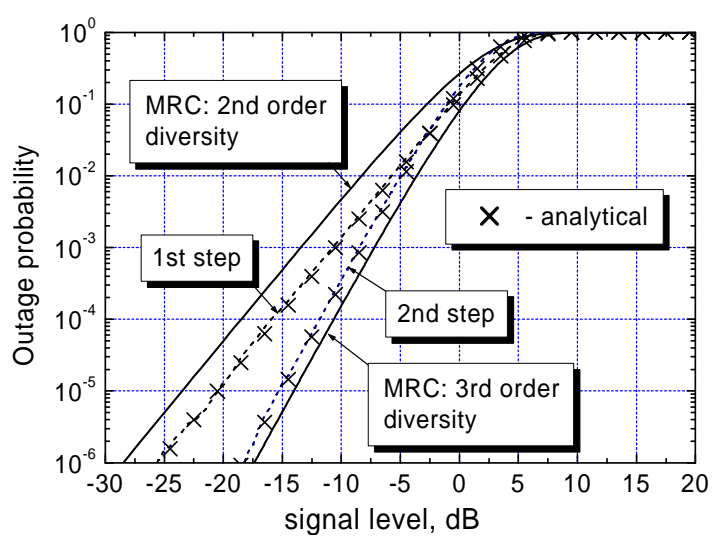

Figure 4. Outage probability curves of the V-BLAST algorithm for $\mathrm{n}=3$.

\section{Numerical MONTE-CARLO Simulations}

In order to validate the analytical results above, we use numerical Monte-Carlo simulations. First, the V-BLAST algorithm outage curves have been simulated without the optimal ordering. No difference has been observed between the analytical results above and the Monte-Carlo simulations (thus, the results are not shown on the graphs), which validates the analytical results. Secondly, the V-BLAST outage curves have been simulated with the optimal ordering procedure. Some of the results are presented in Fig. 3-4. No significant difference has been found between analytical outage probability results and Monte-Carlo simulations. This validates our conclusion that the effect of the optimal ordering is to increase signal power (and SNR) rather than to increase the diversity order.

\section{CONCLUSIONS}

Using a closed-from model of the Gram-Schmidt process, we have developed an analytical approach to the performance analysis of the V-BLAST algorithm. In particular, closed-form analytical expressions have been presented for the signal and noise vectors at $\mathrm{i}$-th processing step, as well as for the outage probabilities.

We have proved that the diversity order at i-th processing step is $(n-m+i)$, provided that no optimal ordering is used. For the $2 \mathrm{xn}$ system, the effect of the optimal ordering at the $1^{\text {st }}$ detection step is to increase SNR by $3 \mathrm{~dB}$ rather then to increase the diversity order (as one might intuitively expect based on the selection combining argument). For the $2^{\text {nd }}$ detection step, the effect of the optimal ordering is to increase the outage probability twice. This is the "price" to pay for increased SNR at the $1^{\text {st }}$ step. However, the diversity order at the second step is $n$. Thus, $3 \mathrm{~dB}$ increase in outage probability will not degrade the overall performance since the original outage probability is low (for reasonably large SNR). On the contrary, it is important to improve the $1^{\text {st }}$ step SNR since the diversity order is (n-1), less than at the second step.

\section{REFERENCES}

[1] G.J Foschini, 'Layered space-time architecture for wireless communication in a fading environment when using multiple antennas', Bell Lab. Tech. J., vol. 1, N. 2, pp. 41-59, 1996.

[2] Foschini, G.J., Gans M.J.: 'On Limits of Wireless Communications in a Fading Environment when Using Multiple Antennas', Wireless Personal Communications, vol. 6, No. 3, pp. 311-335, March 1998

[3] I.E. Telatar, "Capacity of Multi-Antenna Gaussian Channels," AT\&T Bell Lab. Internal Tech. Memo., June 1995 (European Trans. Telecom., v.10, N.6, Dec.1999)

[4] G.D. Golden, G.J. Foschini, R.A. Valenzuela, P.W. Wolniansky, 'Detection Algorithm and Initial Laboratory Results Using V-BLAST Space-Time Communication Architecture', Electronics Letters, vol. 35, No. 1, pp.14-16, $7^{\text {th }}$ January 1999.

[5] G.J Foschini et al, Simplified Processing for High Spectral Efficiency Wireless Communication Employing Multi-Element Arrays, IEEE Journal on Selected Areas in Communications, v. 17, N. 11, pp. 18411852, Nov. 1999

[6] S. Loyka, F. Gagnon, Performance Analysis of the V-BLAST Algorithm: An Analytical Approach, 2002 International Zurich Seminar on Broadband Communications, Feb. 18-21, Zurich, Switzerland.

[7] F.R. Gantmaher, Theory of Matrices, Nauka, Moscow, 1988 (in Russian). 